\title{
A NEW METHOD FOR 3D SHAPE INDEXING AND RETRIEVAL IN LARGE DATABASE BY USING THE LEVEL CUT
}

\author{
M. Elkhal, A. Lakehal and K. Satori \\ Department of Computer Science, Faculty of Science Dhar El Marhaz, LIIAN, \\ Sidi Mohamed Ben Abdallah University, B.P 1796 Fez, Morocco
}

Received 2014-03-30; Revised 2014-04-03; Accepted 2014-05-22

\begin{abstract}
In this study, we propose a new method for indexing and retrieval of 3D models in large databases based on binary images extracted from the 3D object called "level cut" LC. These cuts are obtained by the intersection of the set of the plans with the 3D object. A set of equidistant parallel plans generates by the intersection with the 3D object a set of cuts that used to indexing the 3D model. We are based on these cuts to describe the 3D object by using the vectors descriptors based on these cuts. To validate our descriptor we extract a test database from the NTU base. The robustness of our descriptor is well demonstrated by the comparison with the two will known descriptors, the 3D Zernike descriptor and the 3D invariant moment's descriptor. The topological problem of the external surfaces that representing the 3D object has been confronted, that shows the superiority of the cut method, because it is keeping the external surfaces details of the 3D object during the cutting step.
\end{abstract}

Keywords: Characteristics Level Cut, Vector Descriptor, 3D Shape Indexing and Retrieval, X-means Algorithm, Similarity Measuring

\section{INTROCUCTION}

Recently, the technological development of 3D modeling tools is forever exploited. Many 3D models are now downloadable for free thanks to the internet, as well as the large databases of 3D models have become available in the market. The retrieval of these models in databases demands sophisticated methods for a rapid and effective response.

Among the indexation approaches of 3D models, we find the 2D/3D approach (Haris et al., 2014; Jain and Singh, 2013; Li and Johan, 2013; Petre et al., 2010) which is based on a set of images (the characteristic views, images of depths, the slices...). The importance of this approach is extract for each image a descriptor vector for construct a set of vectors descriptors associated with the object to index. The similarity measure between two 3D objects then returns to calculate the distance between the two sets of descriptors representing them. The extraction of information of the 3D object in a robust and efficient way Recently, the technological development of 3D has become a real challenge. The proposed method permits to deal with this problem. Given a 3D object, the method starts by a normalization step of the processed object using the CPCA technique. Then, we extract a set of cuts based on our cutting method. This set of cuts (binary images) will be indexed by a $2 \mathrm{D}$ image descriptor to construct the descriptor vector associated to the $3 \mathrm{D}$ model, using the Hausdorff distance to calculate the distance between the vectors descriptors which allows us to measure the similarity between the 3D objects.

The proposed descriptor shows the robustness in terms of response to the query provided by the user and the comparative study between the proposed descriptor and two other descriptors well known of the 3D/3D approach, the 3D Zernike moments and 3D invariant moments shows considerable results.

Corresponding Author: M. Elkhal, Department of Computer Science, Faculty of Science Dhar El Marhaz LIIAN, Sidi Mohamed Ben Abdallah University, B.P 1796 Fez, Morocco 
This study will be organized as follows: In the first part, there will be an overview on previous work of 2D/3D approach. After that, we explain the method we proposed in the second part. Then the experiments and results of our method will be shown in the third part and finally the conclusion of our work.

\section{PREVIOUS WORKS}

\subsection{Approach Based Views}

The principal objective of the characteristic views is associated to the $3 \mathrm{D}$ model a series of multiple projections of viewpoints. This principal is common to all the $2 \mathrm{D} / 3 \mathrm{D}$ approaches by view. The problem of this approach is in the choice of: Normalization of the pose, the projection area, the size of image, the nature of the $2 \mathrm{D}$ shape descriptors and the size of the signature. The authors (Ricard et al., 2005; Ansary et al., 2005; Mahmoudi and Daoudi, 2002; Nidhal and Lamis, 2014) have proposed methods of optimal selection of $2 \mathrm{D}$ views to represent a 3D model. Each 3D object is represented by a view set called the characteristic views. The process of selection of views characteristics is based on an adaptive classification algorithm to select the optimal number of views which are calculated from different points of view. These views are represented on the bounding sphere of the $3 \mathrm{D}$ object. In (Cyr and Kimia, 2001), each object is associated to 72 views which are structured in a shock graph. The shape retrieval process is based on a shock graph matching algorithm. The major drawback of the method by views is facing the problems of views choice which do not permit to have adaptive methods to the object and the query.

\subsection{Approach Based on Slices}

Other approaches based on the Cut constructed from 3D objects. (Pu et al., 2004) Represented a 3D object by a set of $2 \mathrm{D}$ cut (slice) taken along the directions of the coordinate system. They consider two intrinsic marks to the 3D model. The first defined by the principal directions and the second by the directions of maximum orientation and choose between the two the one minimizing the volume of the bounding box and used the $2 \mathrm{D}$ shape descriptor to characterize each cut and the distribution of the distance between two points taken at random.

\section{PROPOSED METHOD}

\subsection{The Level Cuts}

The Triangle meshes that we will use frequently constitute the support of representation of the 3D objects. they are represented the major part of the quantity of information describing the $3 \mathrm{D}$ model and are more efficient in the calculation, less expensive and able to represent the $3 \mathrm{D}$ object faithfully. Our method consist to extract a set of binary images called Level Cuts (LC) obtained by the intersection of the 3D object with a set of well defined plans. These images used for indexing it and we can construct the 3D object it from these images set. In what follows we are going to give the steps of the LC set extraction.

\subsection{Ray-Facet Intersection}

Given a triangle $\mathrm{PQR}$ and an $\mathrm{O}$ origin ray and direction vector $\vec{v}$ as shown in the Fig. 1 below:

Let $\mathrm{M}$ the point obtained by the intersection of the ray directed by the $\vec{v}$ vector and the triangle. This point is given by the following relation:

$\overrightarrow{\mathrm{OM}}=\mathrm{d} \cdot \overrightarrow{\mathrm{v}}$

Knowing that $\mathrm{M}$ verified the following Equation 2:

$\overrightarrow{\mathrm{OP}} \cdot \overrightarrow{\mathrm{n}}=\overrightarrow{\mathrm{OM}} \cdot \overrightarrow{\mathrm{n}}$

where, $\overrightarrow{\mathrm{n}}$ that the normal vector to the facet PQR (direct orientation) defined by Equation 3:

$$
\overrightarrow{\mathrm{n}}=\frac{\overrightarrow{\mathrm{PQ}} \wedge \overrightarrow{\mathrm{PR}}}{\|\overrightarrow{\mathrm{PQ}} \wedge \overrightarrow{\mathrm{PR}}\|}
$$

The Equation 1 permits to calculate the distance $d$ between the ray origin and the $\mathrm{M}$ point obtained by intersection with this ray and the surface delimited by the $\mathrm{PQR}$ triangle. To verify that the intersection of ray and the triangle is not empty, i.e., the point $\mathrm{M}$ is in the delimited surface by the PQR triangle, it is enough that the M point should check the following conditions Equation 4:

$$
\begin{aligned}
& (\overrightarrow{M P} \wedge \overrightarrow{M Q}) \cdot \vec{n}>0,(\overrightarrow{M Q} \wedge \overrightarrow{M R}) \cdot \vec{n}>0 \\
& (\overrightarrow{M R} \wedge \overrightarrow{M P}) \cdot \vec{n}>0
\end{aligned}
$$

\subsection{Level Cut Extraction}

These preliminary treatments are necessary if we want to index the 3D object, we base on these cuts. To get the cut in a given direction, we move the ray in the associated plan. The Fig. 2 below shows the extraction of the level cut $\mathrm{y}=\mathrm{k}$ (plan equation $\mathrm{y}=\mathrm{k}$ ) in which the ray (color red) moves in the direction $\overrightarrow{\mathrm{ox}}$. 


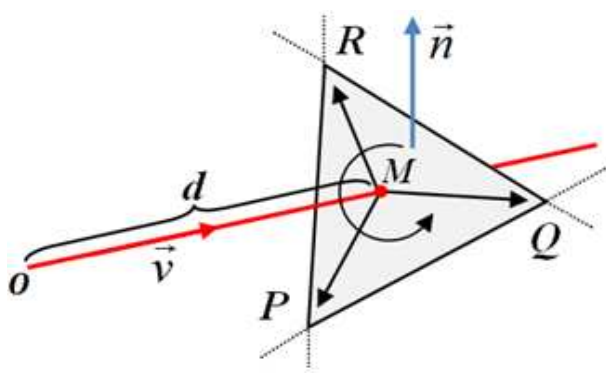

Fig. 1. Ray-facet intersection
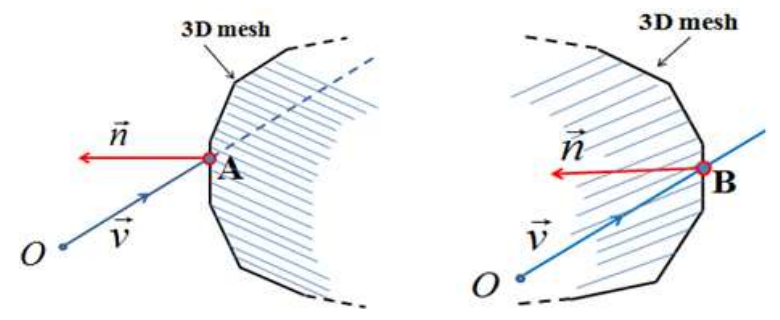

Fig. 2. Test of normal ray with the normal 3D object. A: Incoming point belongs to $\mathrm{g}_{\text {Rin }} \mathrm{B}$ : Outgoing point belongs to $\mathrm{g}_{\text {Rout }}$

The set of the ray intersection points noted $(\Delta)$ with the surface of the $3 \mathrm{D}$ object $\mathrm{O}$ is defined by the following relation Equation 5:

$\mathrm{g}=\Delta \bigcap \mathrm{O}=\mathrm{g}_{\text {Rin }} \mathrm{Ug}_{\text {Rout }}$

Knowing that is the subset of $g$ containing the triangles associated with points whose normal vector is outgoing which is reflected by the following relation in the Fig. 2 Equation 6:

$\vec{n} . \vec{v}>0$

The same for the $\mathrm{g}_{\text {Rout }}$ set which is associated to the triangles witch the normal vector is incoming checked by the following relation Equation 7:

$\vec{n} . \vec{v}<0$

The example in Fig. 3 shows the launch of a ray in the plan $\mathrm{z}=\mathrm{k}$ in the OY direction. The two points $\mathrm{P}_{\mathrm{in}}^{1}$ and $\mathrm{P}_{\text {in }}^{2}$ belong to $\mathrm{g}_{\text {Rin }}$ set and the two points $\mathrm{P}_{\text {out }}^{1}$ and $\mathrm{P}_{\text {out }}^{2}$ belong to the $\mathrm{g}_{\text {Rout }}$ set. The displacement of ray in the plan defined by the $\mathrm{z}=\mathrm{k}$ equation in the $\mathrm{OY}$ direction, generates a set of intersection points of this ray with the $3 \mathrm{D}$ object. Each incoming point of $g_{\text {Rin }}$ set corresponds to an outgoing point of $g_{\text {Rout }}$ set. The segment connecting these two points is a part of the image constructed by the cut. This algorithm is applied to each pair of the set Equation 8: $\mathrm{g}_{\text {Rin }} \times \mathrm{g}_{\text {Rout }}$

Given a 3D object aligned as shows in Fig. 4.

\subsection{The 3D Mesh Rigularity}

The considered triangular meshes surfaces must comply with certain regularity hypothesis. We are going to introduce some definitions to precise the context mathematically. Each facet of the 3D mesh is oriented according to the normal vector which it orthogonal and whose the direction is given by the order of course of the vertices as the example shown in Fig. 4. A mesh is said adjustable if all facets have the coherent direction in Fig. 4a, i.e., they are oriented in the same direction and the edge common $(\mathrm{s} 1, \mathrm{~s} 2)$ to two avoisines facets are going in the same direction. The Fig. $\mathbf{4 b}$ had shown an example of non-coherent mesh by the opposite directions.

\subsection{Problem Found and Proposed Solution}

Before completing the segments between the points obtained by the intersection of the ray with the $3 \mathrm{D}$ object, this will lead us to process particular cases to determine the points accept a segment. The example in the Fig. 5 Shows the problem solved of the ray intersection with the 3D object in corners $P_{(\text {in,out })}$, such as each corner accept two points $P_{\text {Rin }}$ and $P_{\text {Rout }}$ at the same time. The interest to tackle this problem is a detected result because of tTo extract the level cut of a 3D object, this latter must submit a preliminary step of normalization as well as its alignment. he complexity of 3D objects, which sometimes know hundreds of miles of faces and vertices.

\subsection{The Discretization Method of the Cut}

The principle of cuts extraction needs a discretization method for transform the obtained analog information into digital information. The cut is encoded into memory with a $2 \mathrm{D}$ array whose data contains digital values that will be reflected in the colors of pixels on the screen, in our case the cut is black and white where a white pixel is coded with a 1 and a black pixel with a 0 . The resolution is therefore the link between the number of pixels of an image and its actual size on a physical support. The extraction procedure of level cuts follows a step of translation the S set of scatter plots associated with the 3D object towards the positive parts of the mark, which result in the following transformations Equation 9:

$$
\begin{aligned}
\mathrm{x}_{\mathrm{tr}}^{\mathrm{p}} & =\mathrm{x}^{\mathrm{p}}-\mathrm{x}_{\min } \\
\forall \mathrm{p} \in \mathrm{S}, \mathrm{y}_{\mathrm{tr}}^{\mathrm{p}} & =\mathrm{y}^{\mathrm{p}}-\mathrm{y}_{\min } \\
\mathrm{z}_{\mathrm{tr}}^{\mathrm{p}} & =\mathrm{z}^{\mathrm{p}}-\mathrm{z}_{\min }
\end{aligned}
$$




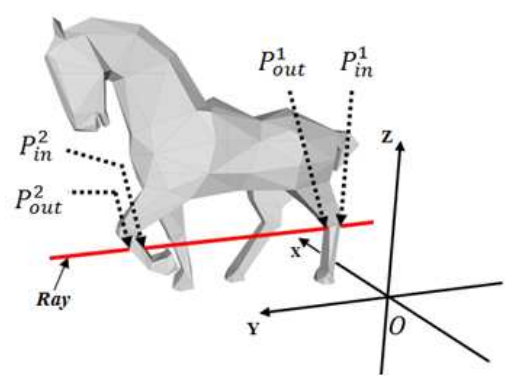

Fig. 3. Ray intersection with the $3 \mathrm{D}$ object
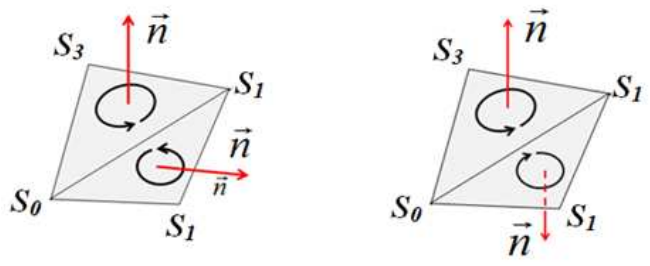

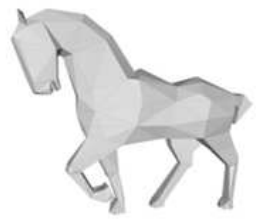

(a)

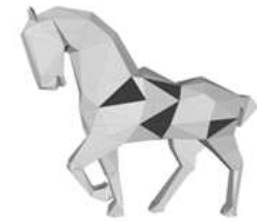

(b)
Fig. 4. (a) coherent Object, (b) non-coherent Object

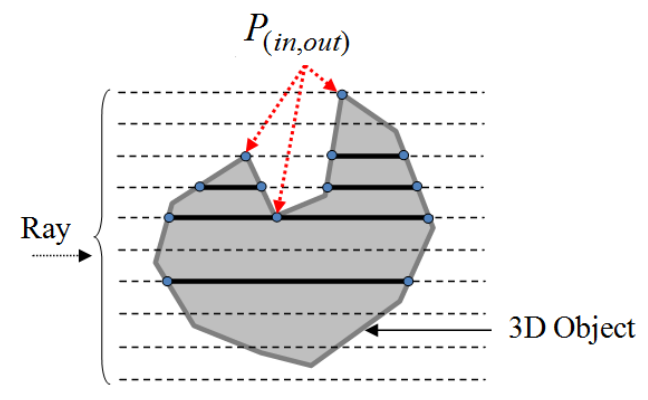

Fig. 5. Case of points accept a segment

With $\mathrm{x}_{\min }, \mathrm{y}_{\min }$ and $\mathrm{z}_{\min }$ the coordinates of extreme points of $\mathrm{S}$ along the axes ox, oy, oz respectively defined by Equation 10:

$$
\begin{aligned}
& \mathrm{x}_{\text {min }}=\underset{\mathrm{x}}{\operatorname{argmin}}\{\mathrm{p}(\mathrm{x}, \mathrm{y}, \mathrm{z}) \in \mathrm{S}\} \\
& \mathrm{y}_{\text {min }}=\underset{\mathrm{y}}{\operatorname{argmin}}\{\mathrm{p}(\mathrm{x}, \mathrm{y}, \mathrm{z}) \in \mathrm{S}\} \\
& \mathrm{z}_{\text {min }}=\underset{\mathrm{z}}{\operatorname{argmin}}\{\mathrm{p}(\mathrm{x}, \mathrm{y}, \mathrm{z}) \in S\}
\end{aligned}
$$

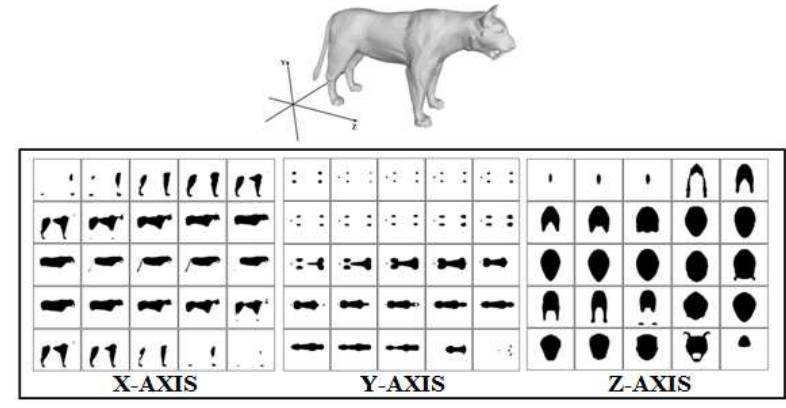

Fig. 6. The level cuts extraction result

The next step is to extract all of the level cut set constructed by the intersection of the $3 \mathrm{D}$ object with a set of parallel planes $\Delta_{\mathrm{K}}$ equidistant by the distance $\delta_{\mathrm{t}}$ with $\mathrm{t} \in\{\mathrm{x}, \mathrm{y}, \mathrm{z}\}$ knowing that Equation 11:

$\mathrm{I}_{\mathrm{K}}=\left(\Delta_{\mathrm{K}}\right) \cap \Theta$

The level cut $\mathrm{k}$ obtained by the intersection of the $3 \mathrm{D}$ object $\Theta$ and the $\Delta_{\mathrm{K}}$ plan according to one of the three directions $\overrightarrow{\mathrm{ox}}, \overrightarrow{\mathrm{oy}}, \overrightarrow{\mathrm{or}} \overrightarrow{\mathrm{oz}}$ with $\delta_{\mathrm{t}}=\frac{\mathrm{t}_{\max }}{\mathrm{n}}, \mathrm{t} \in\{\mathrm{x}, \mathrm{y}, \mathrm{z}\} \mathrm{n}$ The number of cuts to extract.

The example below shows the performance and quality of our experience concerning the part applied on different axes.

Our level cuts extraction result above Fig. 6 shows the accuracy of the quality of the images obtained and the cases that we processed removed the difficulty and problems of density the points in specific parts. This gives us a simplification to process all $3 \mathrm{D}$ objects without any problems.

\section{THE DESCRIPTOR BASED ON THE CLC SET AND THE SIMILARITY MEASURING}

\subsection{The Descriptor Based on the LC Set}

The descriptor that we proposed above is based on binary images set called Characteristics Level Cut to indexing the $3 \mathrm{D}$ model by using the following steps:

- Normalize the 3D model

- The LC set extraction

- Reduce of the LC set by using the X-means algorithm to extract the CLC set

- Extraction of the signature for each binary image to constructing the vector descriptor of the 3D model 


\subsection{Normalisation of 3D Model}

The 3D object is often found in an arbitrary position (scale, position and orientation), which requires a pretreatment step before extracting the vectors descriptors. First, we must make this object invariant to scale space and then using the ACPC (Vranic et al., 2001) method to make the $3 \mathrm{D}$ model invariant in the orientation.

\subsection{The LC Set Extraction}

After the step of normalization, the extraction of level cuts sets follows. Often the topology of the surface representing the $3 \mathrm{D}$ model contains a lot of information, for this reason, we must extract an important number of cuts to better describe the $3 \mathrm{D}$ object as a whole. This number results from the complexity of the $3 \mathrm{D}$ object in the precise parts.

\subsection{Reduction of LC Set Using the X-Means Algorithm}

The increase in the cut number LC is often important for a perfect and accurate description of the $3 \mathrm{D}$ object. Unfortunately, this number actually generates of redundant information as shown in Fig. 7.

To avoid this problem, we used the X-means algorithm to classify similar images in a single class. We took a representative for each ways of these classes.
These representative constructed a new set called the Characteristics Level Cut set (CLC). The Fig. 8 below shows the CLC set of the LC set in (Fig. 7).

\subsection{Vectors Descriptors Extraction}

To construct the vectors descriptors of the $3 \mathrm{D}$ model, using a binary image 2D descriptor to extract the signature of each cut of the CLC set. In our case we will use the seven moment's invariants $\mathrm{Hu}$ (Sabhara et al., 2013), these signatures constructed a set of vectors descriptors associated with the 3D object.

\subsection{Similarity Measuring}

In order to measure how similar two objects are, it is necessary to compute distances between pairs of their vectors descriptors using a dissimilarity measure. As we are using the X-means to extracting the CLC set, the vectors descriptors do not have the same size for each model, so the Euclidean distance is not valid for our method. There are two distances that could adapt with our descriptors, the hausdorff distance and the Earth Mover Distance (EMD) (Rubner et al., 2000; Muruganathan et al., 2014; Edy et al., 2014). The EMD seems very expensive in terms of computation, then hausdorff distance is the most adaptable with the proposed vectors descriptors, also, it is the most used in this kind of problem.

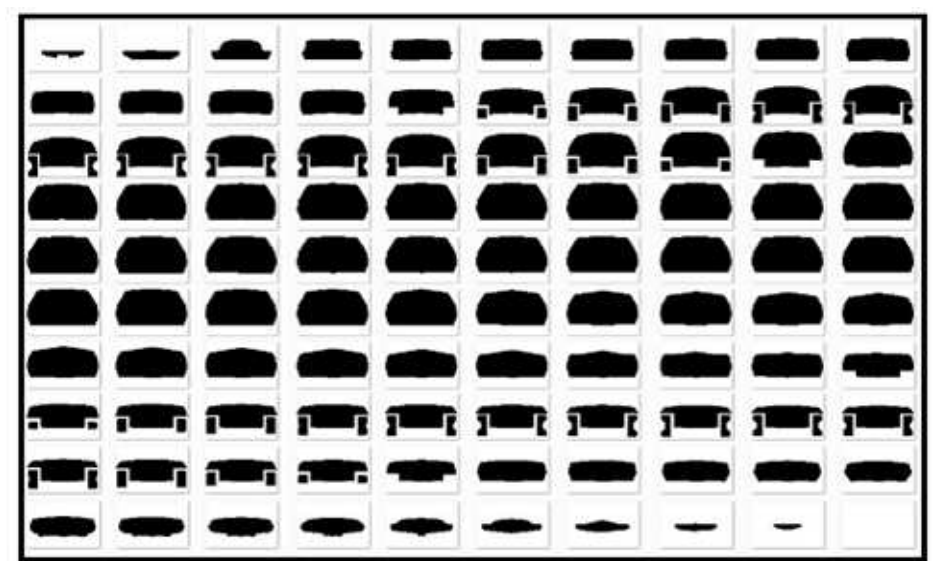

Fig. 7. Extraction of 100 LC set

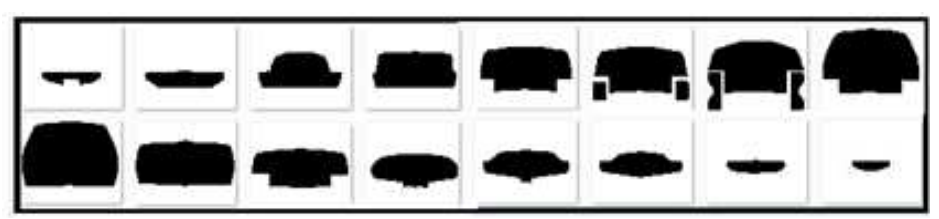

Fig. 8. CLC set obtained by X-means 


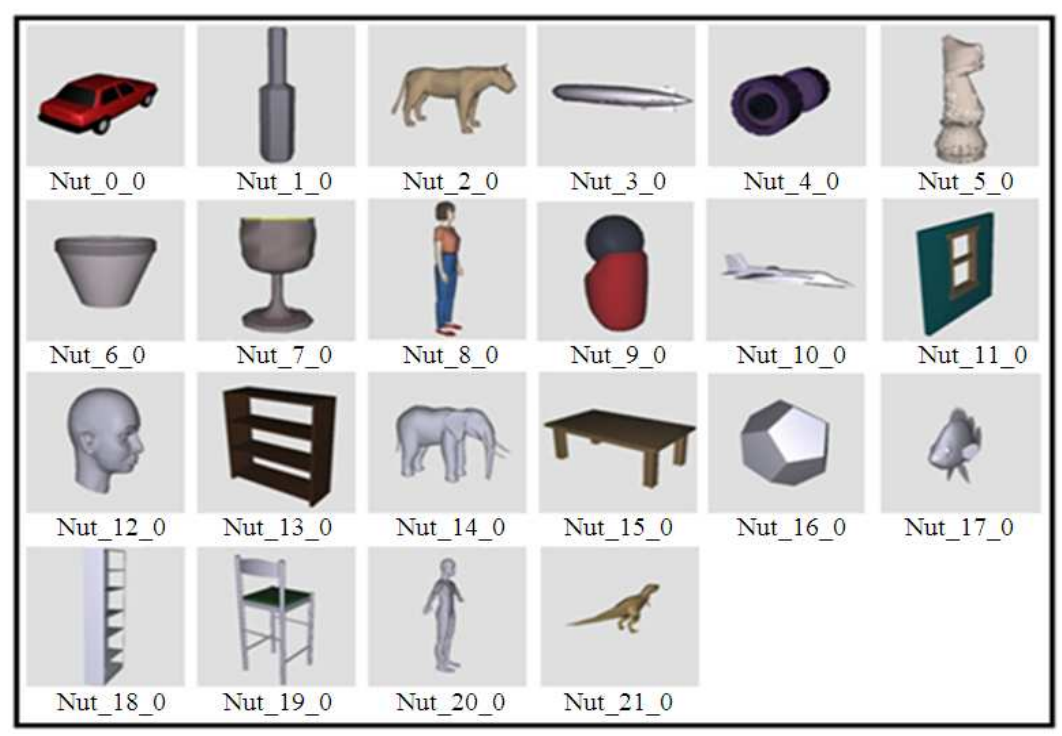

Fig. 9. The representatives of the test database classes

The Hausdorff distance between two no empty set $A$ and $B$ noted $d_{H}(A, B)$ Equation 1 , is the maximum of $\mathrm{H}(\mathrm{A}, \mathrm{B})$ and $(\mathrm{B}, \mathrm{A})$. Thus, it measures the degree of mismatch between two sets by measuring the distance of the point of $A$ that is farthest from any point of $B$ and vice versa. Intuitively, every point of A must be within a distance $\mathrm{d}_{\mathrm{H}}(\mathrm{A}, \mathrm{B})$ of some point of $B$ and vice versa. Thus, the notion of resemblance encoded by this distance is that each member of A be near some member of $\mathrm{B}$ and vice versa. Unlike most methods of comparing vectors, there is no explicit pairing of points of $A$ with points of $B$ (for $B$ ). The function $\mathrm{d}_{\mathrm{H}}(\mathrm{A}, \mathrm{B})$ can be trivially computed in time $\mathrm{O}(\mathrm{pq})$ for two point's sets of size $\mathrm{p}$ and $\mathrm{q}$, respectively and this can be improved to $\mathrm{O}((\mathrm{p}+\mathrm{q}) \log (\mathrm{p}+\mathrm{q}))$ Equation 12:

$$
\begin{aligned}
& d_{H}(A, B)=\max \{H(A, B), H(B, A)\} \\
& =\max \{\max \operatorname{mind}(a, b), \operatorname{maxmind}(a, b)\}
\end{aligned}
$$

where, $\mathrm{d}$ is the Euclidean distance.

Let $\mathrm{M}$ and $\mathrm{Q}$ be two models of the database, their vectors descriptors are defined by $\mathrm{X}^{\mathrm{M}}=\left\{\mathrm{X}_{1}^{\mathrm{M}}, \ldots, \mathrm{X}_{\mathrm{K}}^{\mathrm{M}}\right\}$ and $\mathrm{X}^{\mathrm{Q}}=\left\{\mathrm{X}_{1}^{\mathrm{Q}}, \ldots, \mathrm{X}_{\mathrm{K}}^{\mathrm{Q}}\right\} \quad$ respectively, where $\mathrm{K}$ and $\mathrm{h}$ represent the number of elements of LI set of the models $\mathrm{M}$ and $\mathrm{Q}$ respectively. The similarity between $\mathrm{M}$ and $\mathrm{Q}$ is measured as follow Equation 13:

$$
\operatorname{Sim}(M, Q)=\frac{1}{1+d_{h}\left(X^{M}, Y^{Q}\right)}
$$

where, $d_{h}\left(X^{M}, X^{Q}\right)$ is defined as Equation 14:

$$
\begin{aligned}
& \mathrm{d}_{\mathrm{h}}\left(\mathrm{X}^{\mathrm{M}}, \mathrm{X}^{\mathrm{Q}}\right)=\max \left\{\operatorname{maxmind}\left(\mathrm{X}_{\mathrm{i}}^{\mathrm{M}}, \mathrm{X}_{\mathrm{j}}^{\mathrm{Q}}\right) ;\right. \\
& \left.\operatorname{maxmind}\left(\mathrm{X}_{\mathrm{i}}^{\mathrm{M}}, X_{\mathrm{j}}^{\mathrm{Q}}\right)\right\}
\end{aligned}
$$

This similarity function (13) has value in the range $[0,1]$, if its value is close to 1 for two models, they are assumed to be similar if it is close to 0 , they are considered dissimilar.

\section{EXPERIMENTS AND RESULTS}

\subsection{Test Database}

Concerning the experimental side we constructed a test database from the NTU base (National Taiwan University) which offers 10,910 3D models of different kinds. The extracted database is divided into 22 classes which include 133 3D models. Each class contains a number of objects as shown in the following Table 1.

The tests were carried on a computer running Windows 7, with 2GB RAM and a $2 \mathrm{GHz}$ Intel processor using a Java platform. In order to evaluate the performance of the shape similarity measure, we design experiments performing 3D model retrievals on the test database. The first model on the left is considered a query as the example shown in Fig. 10. 


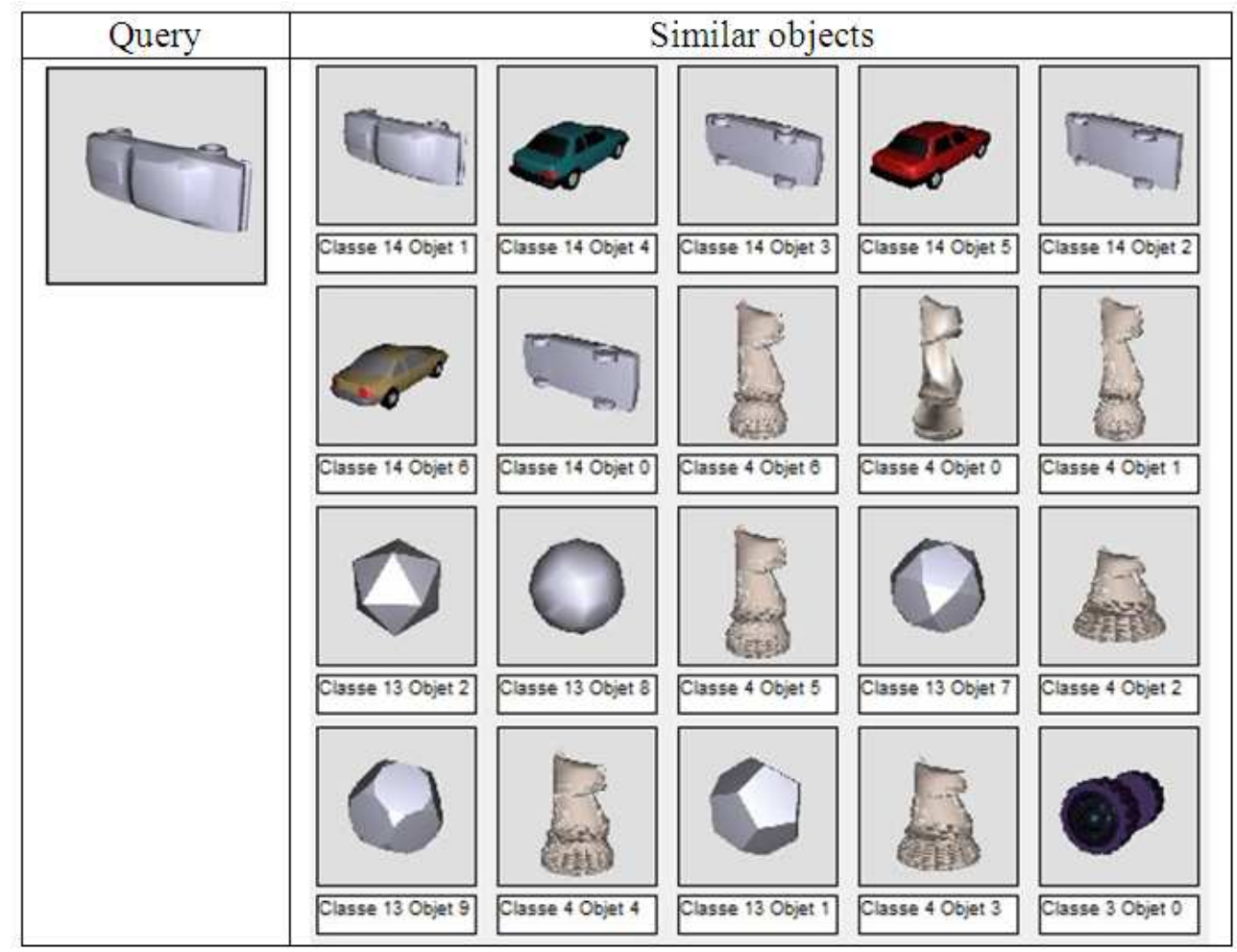

Fig. 10. First twenty shapes retrievals using the CLC set

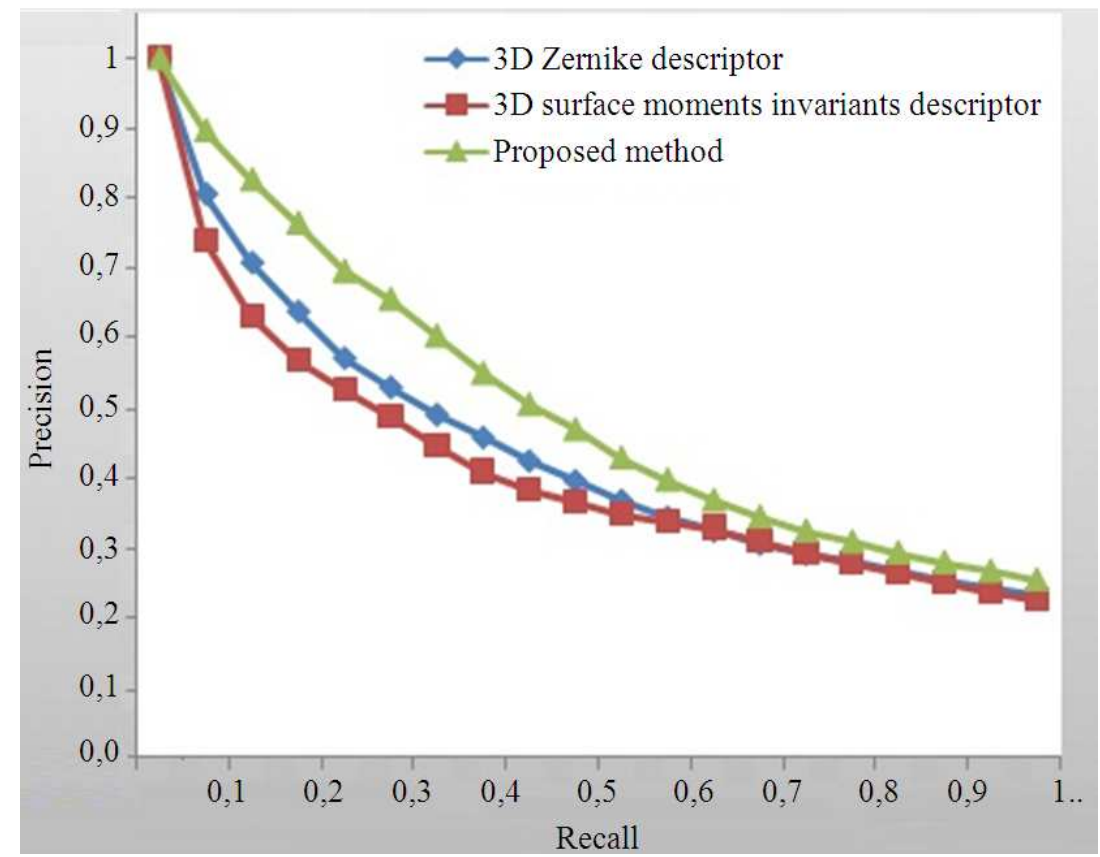

Fig. 11. Precision-Recall graph for all three descriptors 
Table 1. Number object for each class

\begin{tabular}{|c|c|c|c|c|c|c|c|c|c|c|c|}
\hline Class name & 0 & 1 & 2 & 3 & 4 & 5 & 6 & 7 & 8 & 9 & 10 \\
\hline Object number for each class & 10 & 8 & 4 & 6 & 8 & 4 & 4 & 6 & 6 & 4 & 3 \\
\hline class name & 11 & 12 & 13 & 14 & 15 & 16 & 17 & 18 & 19 & 20 & 21 \\
\hline Object number for each class & 8 & 4 & 10 & 7 & 6 & 8 & 6 & 4 & 3 & 8 & 4 \\
\hline
\end{tabular}

Concerning the representatives for each class we have cited them in the Fig. 9 below.

\subsection{Result and Performance}

In order to evaluate the measurement of retrieval performance, we examine the Recall-Precision graph for the shape descriptor proposed. "Precision" Equation15 measures that the ability of the system to retrieve only models that are relevant, while "recall" Equation16 measures the ability of the system to retrieve all models that are relevant. They are defined as :

$$
\begin{gathered}
\text { Recall }=\frac{\text { relevant } \text { correctly retrieved }}{\text { all relevant }} \\
\text { Precesion }=\frac{\text { relevant correctly retrieved }}{\text { all relevants }}
\end{gathered}
$$

We compared the classification performance of the proposed method with two well known methods, that the descriptor of 3D Zernike moments (Sit et al., 2013) and descriptor of moments invariants 3D (Suk and Flusser, 2011). For each one of the chosen shape categories, we have calculated the average Recall-Precision graph by using all shapes of the test database by a query object Fig. 11. We can see that the proposed shape descriptors perform better than the 3D Zernike descriptor and surface moments invariants descriptor.

\section{CONCLUSION}

In this study, we have presented a new set of binary image called Characteristics Level Cut to represent the $3 \mathrm{D}$ model, this set is used to extract the vectors descriptors by using the Hu moment $2 \mathrm{D}$ to indexing each image of this set. The similarity between models is calculated by using the Hausdorff distance. The obtained results show that the proposed descriptor is robust by the comparison with the two known methods which are mentioned above, Despite this, some areas in the 3D object pose again the problems during of the extraction which also requires an improvement, additionally, the content of the image extracted can be contour based or region based, as well as, we can reconstruct the original $3 \mathrm{D}$ object from the set of these images extracted.

\section{REFERENCES}

Ansary, T.F., M. Daoudi and J.P. Vandeborre, 2005. 3D model retrieval based on adaptive views clustering. Proceedings of the 3rd International Conference on Advances in Pattern Recognition, Aug. 22-25, Springer Berlin Heidelberg, Bath, United Kingdom, pp: 473-483. DOI: 10.1007/11552499_53

Cyr, C. and B. Kimia, 2001. 3D object recognition using shape similarity-based aspect graph. Proceedings of the International Conference on Computer Vision, Jun. 7-14, IEEE Xplore Press, Vancouver, BC., pp: 254-261. DOI: 10.1109/ICCV.2001.937526

Edy, W., H. Agus, A.M. Arymurthy and E. Winarko, 2014. Improved real-time face recognition based on three level wavelet decomposition-principal component analysis and mahalanobis distance. J. Comput. Sci., 10: 844-851. DOI: 10.3844/jcssp.2014.844.851

Haris, A., H. Rangkuti, A. Harjoko and A. E. Putro, 2014. Content based batik image retrieval. J. Comput. Sci., 10: 925-934. DOI: 10.3844/jcssp.2014.925.934

Jain, Y.K. and R.K. Singh, 2013. Efficient view based 3-D object retrieval using hidden markov model. Depart. Comput. Sci. Eng., 4: 1-7. DOI: 10.1007/3DRes.04(2013)5

Li, B. and H. Johan, 2013. Sketch-based 3D model retrieval by incorporating 2D-3D alignment. School Comput. Eng., 65: 363-385. DOI: 10.1007/s11042-012-1009-0

Mahmoudi, S. and M. Daoudi, 2002. 3D models retrieval by using characteristic views. Proceedings of the 16th International Conference on Pattern Recognition, Aug. 11-15, IEEEE Xplore Press, pp: 11-15. DOI: 10.1109/ICPR.2002.1048337

Muruganathan, S., N. Devarajan, D. Chitra and T. Manigandan, 2014. Shape retrieval through mahalanobis distance with shortest augmenting path algorithm. J. Comput. Sci., 10: 552-562. 10.3844/jcssp.2014.552.562 
Nidhal, K.E. and L. Al Saadi, 2014. Fast image matching in huge database. J. Comput. Sci., 10: 1488-1496. DOI: $10.3844 /$ jcssp.2014.1488.1496

Petre, R.D., T. Zaharia and F. Prêteux, 2010. An overview of view-based 2D/3D indexing methods. Proceedings of the Mathematics of Data/Image Coding, Compression and Encryption with Applications, Aug. 23-23, SPIE, San Diego, California, pp: 12-12. DOI: 10.1117/12.861542

$\mathrm{Pu}$, J., Y. Liu, X. Gu, W. Liu and H. Zha et al., 2004. 3D model retrieval based on $2 \mathrm{D}$ slice similarity measurements. Proceedings of the 2nd International Symposium on 3D Data Processing, Visualization and Transmission, Sept. 6-9, IEEE Xplore Press, pp: 95-101. DOI: 10.1109/TDPVT.2004.1335181

Ricard, J., D.C. Jolly and A. Baskurt, 2005. Generalizations of angular radial transform for $2 \mathrm{D}$ and 3D shape retrieval. Patt. Recogn. Lett., 26: 2174-2186. DOI: 10.1016/j.patrec.2005.03.030

Rubner, Y., C. Tomasi and L.G. Guibas, 2000. The earth mover's distance as a metric for image retrieval. Int. J. Comput. Vision, 40: 99-121. DOI: 10.1023/A: 1026543900054
Sabhara, R.K., C. Lee and K. Lim, 2013. Comparative study of hu moments and zernike moments in object recognition. Smart Comput. Rev., 3: 166-173. DOI: 10.6029/smartcr.2013.03.003

Sit, A., J.C. Mitchell, G.N. Phillips and S.J. Wright, 2013. An extension of 3D zernike moments for shape description and retrieval of maps defined in rectangular solids. Molecular Based Math. Biol., 1: 75-89. DOI: $10.2478 / \mathrm{mlbmb}-2013-0004$

Suk, T. and J. Flusser, 2011. Tensor method for constructing 3D moment invariants. Comput. Anal. Images Patt., 6855: 212-219. DOI: 10.1007/978-3642-23678-5_24

Vranic, D.V., D. Saupe and J. Richter, 2001. Tools for 3D-object retrieval: Karhunen-loeve transform and spherical harmonics. Proceedings of the IEEE 4th Workshop on Multimedia Signal Processing, Oct. 35, IEEE Xplore Press, Cannes, pp: 293-298. DOI: 10.1109/MMSP.2001.962749 